\title{
Aplicações da ressonância magnética para medidas espectroscópicas da neurotransmissão
}

\author{
Graeme F Masona, Kevin L Behar ${ }^{b}$, John H Krystal ${ }^{b}$ e Douglas L Rothman ${ }^{c}$ \\ ${ }^{a}$ Department of Psychiatry of the Yale University, School of Medicine, Haven, USA e Department of Biomedical Engineering of the Yale \\ University, School of Medicine, Haven, USA. bepartments of Psychiatry of the Yale University, School of Medicine, Haven, USA. ${ }^{\mathrm{b}}$ Departments \\ of Diagnostic Radiology of the Yale University, School of Medicine, Haven, USA
}

\section{Introdução}

A ressonância magnética é conhecida pelas imagens anatômicas. É uma técnica de grande utilidade por não usar radiação ionizante e por permitir o estudo de aspectos fisiopatológicos de vários transtornos cerebrais. Com desenvolvimentos recentes na metodologia de espectroscopia por ressonância magnética (ERM), hoje em dia é possível medir substâncias químicas específicas durante tratamentos farmacológicos, ou alterações neuroquímicas que possam estar relacionadas com processos fisiopatológicos presentes em várias doenças neuropsiquiátricas, em estágios iniciais nos quais ainda não se pode observar mudanças anatômicas correspondentes, como, por exemplo alterações nos níveis de ácidos aspártico$\mathrm{N}$-acetil (ANA) e gama-amino-butírico (GABA). A ERM permite avaliar mudanças cinéticas nas vias metabólicas do cérebro resultantes de tais alterações e propicia também maneiras de se medir as velocidades de síntese de vias neuroquímicas e de neurotransmissão nos seres humanos in vivo. O objetivo do presente artigo consiste na descrição do emprego da ERM para tais medidas cinéticas.

A teoria básica da espectroscopia por ressonância magnética

Os núcleos de alguns isótopos do hidrogênio, do fósforo, do carbono, e de alguns outros elementos, têm propriedades quân ticas que lhes fazem se comportar de uma maneira análoga aos ímãs comuns. Na presença de um campo magnético dezenas de milhares vezes maior que a o campo magnético da Terra, podem propiciar uma quantidade considerável de informações químicas.

Dentro do campo magnético, os núcleos respondem com frequiências similares às utilizadas nas faixas de rádios FM, em proporção à magnitude do campo magnético, pois a ressonância clínica de 1,5 Tesla tem uma freqüência de $64 \mathrm{MHz}$, enquanto que uma máquina de 8 Tesla tem uma frequiência de $343 \mathrm{MHz}$. Para adquirir o sinal, são transmitidos pulsos magnéticos de microsegundos a milisegundos, com a freqüência do campo magnético, os quais perturbam o equilíbrio dos núcleos no ímã (Figura 1B). Assim que voltam ao equilíbrio, os núcleos emitem energia quase na mesma freqüência (Figura 1C). Assim, colocando uma pessoa no aparelho de ressonância magnética e criando-se um campo magnético com a frequiência do ${ }^{1} \mathrm{H}$, recebe-se o sinal mostrado na Figura 1D, que representa

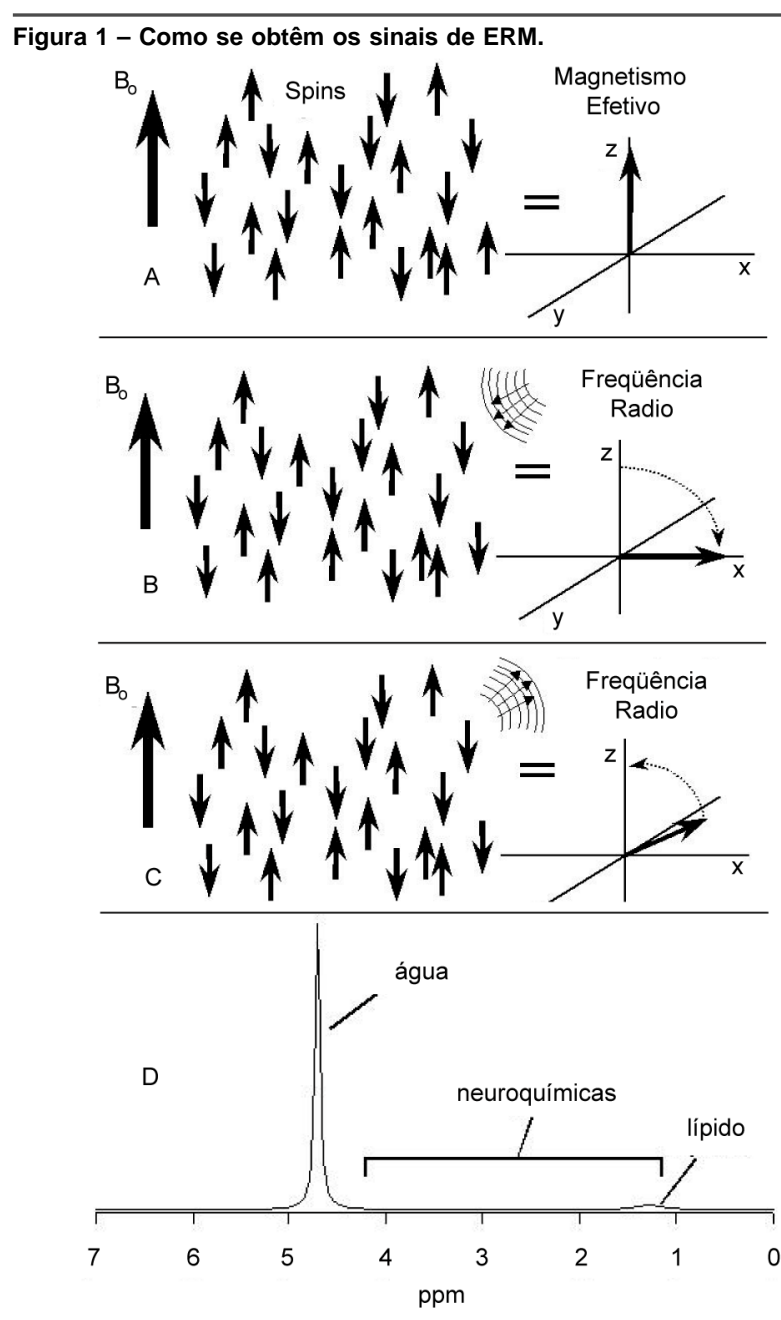

(A) Um cérebro contém muitos "spins" quânticos que se comportam como pequenos ímãs. Colocados num grande campo magnético $B_{\circ}$, uma pequena maioria se orienta na direção do campo, se comportando igual a um vetor. (B) É transmitida energia na freqüência certa para o tamanho do campo, perturbando a distribuição dos spins, resultando num movimento do vetor. (C) Ao voltar ao seu estado de equilíbrio, os spins transmitem um sinal em proporção ao número de núcleos no volume de interesse. (D) O espectro de ERM obtido do cérebro humano mostra os sinais da água e lípides, que ofuscam os sinais de outros compostos neuroquímicos. Existem vários métodos para diminuir os sinais da água e lípides, de modo que se possa quantificar as ressonâncias menores.

principalmente a água, pois a água tem a concentração de ${ }^{1} \mathrm{H}$ de $110 \mathrm{M}$. É esse sinal que se emprega principalmente para fazer as imagens clínicas convencionais da ressonância. 
Em estudos bioquímicos, utiliza-se outra fonte da potência enorme da ressonância: a espectroscopia (ERM). Já foi dito que a freqüência de um núcleo depende da amplitude do campo magnético em volta de si. Embora o maior efeito seja pelo campo principal do ímã, também existe a influência microscópica eletrônica em núcleos atômicos individuais. Por exemplo, os elétrons se aconchegam aos núcleos de influência do campo magnético, pois o oxigênio atrai os elétrons até si mesmo, expondo mais os núcleos de hidrogênio que ficam por perto. Em geral, essas influências aumentam ou diminuem a frequiência de $\mathrm{MHz}$ por uma quantidade de $\mathrm{Hz}$ ou milhares de $\mathrm{Hz}$. Assim, o ácido glutâmico, o neurotransmissor excitatório principal no cérebro, se representa no espectro do hidrogênio em vários locais deste espectro, dependendo da vizinhança local de cada núcleo. Cada composto químico detectado no cérebro humano tem uma distribuição diferente de freqüências (Figura 2), e pelas variações locais se obtém a informação química.

Figura 2 - Espectros típicos adquiridos pela ERM de ${ }^{1} \mathrm{H}$.
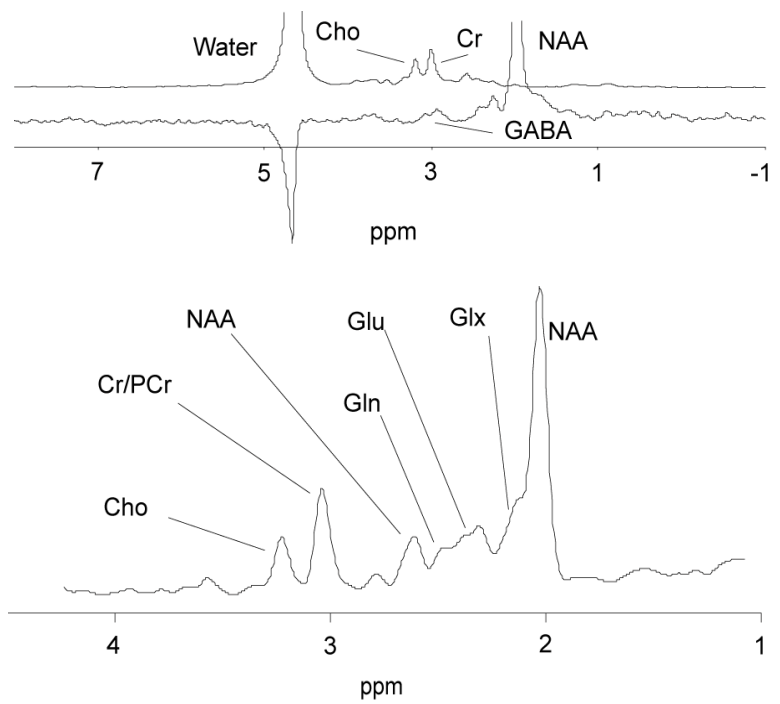

(Acima) Espectros mostrando a creatina (do metabolismo energético), a colina (metabolismo de membrana), o ANA (viabilidade neuronal) e o GABA (neurotransmissor inibitório). (Abaixo) Espectro mostrando a creatina, a colina e o ANA com ácido glutâmico (Glu), a glutamina (Gln) e o ácido aspártico (Asp). O Glu e a Gln também têm uma ressonância combinada correspondendo aos prótons do carbono C3 (Glx), ao lado da ANA.

\section{Aplicações à avaliação da neurotransmissão}

Um dos núcleos até agora mais utilizados na psiquiatria é o ${ }^{1} \mathrm{H}$. Ele é empregado para fazer imagens convencionais de ressonância magnética e para a obtenção de medidas de concentrações de vários metabólitos relacionados com a neurotransmissão. Outro núcleo importante nos estudos da neurotransmissão é o ${ }^{13} \mathrm{C}$, que além de permitir medidas de concentrações metabólicas, serve para medidas cinéticas de metabolismo energético e da neurotransmissão. Outros elementos, tais como o ${ }^{31} \mathrm{P}, \mathrm{o}{ }^{7} \mathrm{Li}$, e o ${ }^{19} \mathrm{~F}$ também são utilizados em estudos relacionados à psiquiatria, mas se prestam a estudos de aspectos diferentes do metabolismo cerebral e ficam fora do foco do presente artigo.

\section{${ }^{1} \boldsymbol{H}$}

A ressonância do ${ }^{1} \mathrm{H}$ tem a grande vantagem da maior sensi- bilidade, devido, em grande parte, ao fato da maioria dos aspectos químicos de interesse serem compostos de muito hidrogênio. Espectros do hidrogênio são vistos na Figura 2, mostrando a detecção do ácido glutâmico, da glutamina, do GABA e de outros compostos neuroquímicos. A maioria dos sinais de interesse se colocam numa região espectral de centenas de $\mathrm{Hz}$, que por razões técnicas facilitam a localização de sinais em volumes específicos. Por outro lado, a proximidade de tantos sinais de interesse introduz o problema da resolução espectral. Isto é, não há resolução suficiente para se quantificar separadamente a glutamina e o ácido glutâmico nos aparelhos de ressonância mais comumente disponíveis para uso humano (1,5 Tesla). A resolução é maior em aparelhos de $2 \mathrm{~T}$ e melhora mais ainda em 4T. ${ }^{1}$ Com a ERM de ${ }^{1} \mathrm{H}$, também tem-se que enfrentar o problema oriundo dos sinais de lípidos e dos $110.000 \mathrm{mM}$ de ${ }^{1} \mathrm{H}$ da água em relação ao sinal de 1-30 mM de ${ }^{1} \mathrm{H}$ dos compostos químicos de interesse. Vários métodos já foram desenvolvidos para diminuição seletiva do sinal oriundo da água presente no tecido cerebral.

Além da água, é possível ver sinais de muitos outros compostos. No presente artigo, os autores concentraram-se em quatro compostos relacionados diretamente à função cerebral: o GABA, o ácido glutâmico, a glutamina e o ANA. O sinal mais destacado do cérebro vem do ANA, que se coloca primariamente em neurônios glutamatérgicos ${ }^{2}$ e tem funções ainda desconhecidas. Logo depois da sua identificação e localização específica em neurônios e seus processos, acreditava-se que uma diminuição do ANA representasse morte neuronal. Porém, estudos mais recentes demonstram a possibilidade da recuperação do ANA, mostrando que basta o comprometimento dos neurônios para diminuir a concentração do ANA. Por exemplo, é possível haver aumento do ANA durante o ciclo menstrual, ${ }^{3}$ ou após o tratamento da epilepsia. ${ }^{4}$ É possível que o nível do ANA se relacione à viabilidade energética do neurônio, pois o ANA é fabricado pela enzima aspartil-acetil transferase, na mitocôndria. A ligação energética abre ainda mais a possibilidade de uma ligação entre o ANA e a função cerebral. Na ERM, demonstra-se reduções do ANA no lobo frontal em pacientes com esquizofrenia, ${ }^{5}$ um transtorno que se manifesta, entre outros sintomas, como déficits da função de regiões frontais. Também é reportada na esquizofrenia uma diferença dos níveis do ácido glutâmico ou da glutamina, ${ }^{6}$ ligando os resultados a alterações neuroquímicas.

O ácido glutâmico e a glutamina podem ser medidos para se observar aspectos funcionais da neurotransmissão. O ácido glutâmico é o neurotransmissor mais comum no cérebro de mamíferos e participa dos ciclos de translocação entre os neurônios e as células astrocíticas ${ }^{7}$ (Figura 3). Resumidamente, os neurônios glutamatérgicos liberam o ácido glutâmico, que é transportado para dentro dos astrócitos, onde se desativa através da conversão em glutamina. A glutamina volta para neurônios glutamatérgicos para reconverter-se em ácido glutâmico e se transloca também ao neurônio GABAérgico, onde se converte no GABA. Outro ciclo químico análogo consiste na entrada do ácido glutâmico nos neurônios GABAérgicos para se converter no GABA. Pode-se, com facilidade, imaginar que modificações 
em quaisquer desses ciclos podem alterar as concentrações do ácido glutâmico, da glutamina e do GABA. Na depressão, são encontradas diminuições do GABA em $30-40 \%$ dos pacientes, em comparação com controles, em áreas do córtex occipital. ${ }^{8,9} \mathrm{~A}$ diminuição do GABA normaliza-se após tratamento com antidepressivos, ${ }^{10}$ mas os efeitos no ácido glutâmico ainda não foram investigados. $\mathrm{O}$ ácido glutâmico está alterado na depressão, mas existe controvérsia se há um aumento ${ }^{9}$ ou uma diminuição. ${ }^{11}$ Também são relatadas alterações do GABA, do ácido glutâmico, da glutamina ou do ANA na detoxificação do álcool, ${ }^{12}$ no transtorno disfórico pré-menstrual, ${ }^{3,13}$ em cocainômanos ${ }^{14}$ e no transtorno do pânico.* Figura 3 - O metabolismo de glicose (GIc) desde o sangue, pela glicólise
(Vglc), até o ciclo neurotransmissor de glutamina (GIn), ácido glutâmico
neuronal e astrocítico $\left(\mathrm{Glu}_{\mathrm{N}}\right.$ e $\left.\mathrm{Glu}_{\mathrm{A}}\right)$.

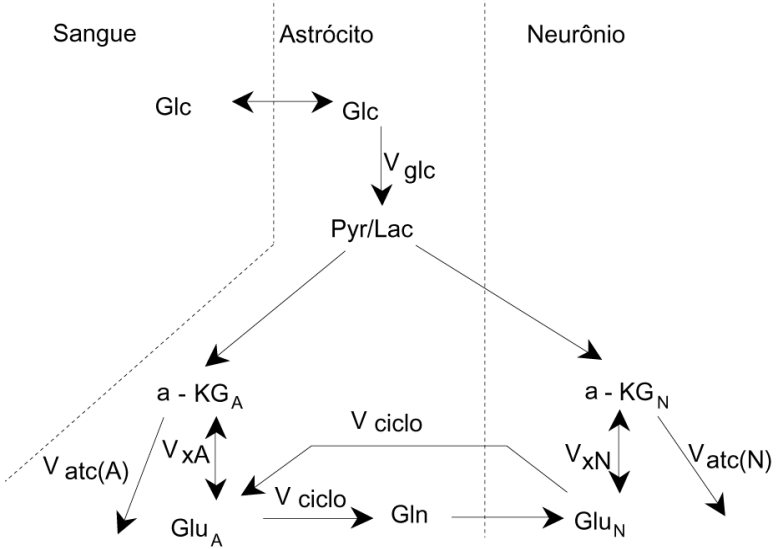

Oneurônio e o astrócito têm um ciclo de ácido tricarboxílico, onde entra o carbono dos ácidos pirúvico e láctico ( $\mathrm{Pyr} / \mathrm{Lac})$, chegando até o ácido alfa-glutárico $(\mathrm{a}-\mathrm{Kg})$, onde um equilíbrio rápido $\mathrm{V}_{\mathrm{xA}} e \mathrm{~V}_{\mathrm{xN}}$ ) transloca o carbono ao Glu. O Glu se solta na neurotransmissão, entra no astrócito e se mistura com o Glu para se converter em glutamina e voltar ao neurônio. Os fluxos são representados por equações matemáticas, com velocidades que se ajustam para se adequar aos representados por equados experimentais.
${ }^{13} \mathrm{C}$

Um por cento do carbono natural é composto pelo isótopo

${ }^{13} \mathrm{C}$, que apesar de não ser radioativo pode ser detectado pela ERM e oferece a possibilidade de interpretar a bioquímica pelas medidas da ERM de ${ }^{1} \mathrm{H}$. O ${ }^{13} \mathrm{C}$ é um isótopo que é detectado com sensibilidade menor que o ${ }^{1} \mathrm{H}$ em estudos com ERM, mas a sua baixa concentração no cérebro propicia a possibilidade de se realizar estudos em que se pode introduzir substratos naturais enriquecidos com ${ }^{13} \mathrm{C}$ e observar o surgimento dos produtos marcados com o ${ }^{13} \mathrm{C}$ no cérebro in vivo (Figura 4A). Em geral, quanto mais rapidamente se detecta esses produtos de vias cerebrais específicas, mais rápidos são os processos metabólicos cerebrais que geram esses produtos. ${ }^{15}$ Até agora, a maioria dos estudos têm utilizado a $\left[1-{ }^{13} \mathrm{C}\right]$ glicose e a [2$\left.{ }^{13} \mathrm{C}\right]$ glicose, utilizando a ERM no cérebro in vivo para detectar o ácido glutâmico e a glutamina, para medir as velocidades de oxidação de glicose ${ }^{16-19}$ o ciclo neurotransmissor entre o ácido glutâmico e a glutamina, ${ }^{20,21}$ as vias anapleróticas ${ }^{22}$ e vários níveis de função. ${ }^{23}$

\section{Emprego do ${ }^{13} \mathrm{C}$ para medidas cinéticas}

Esse tipo de uso da ERM pode ser exemplificado pela situação de um produto $P$ enriquecido pelo isótopo de um substrato $S$ em qual já se incorporou o ${ }^{13} \mathrm{C}$. Nessa situação idealizada, com o enriquecimento do $S$ subindo no momento $t=0$, imediatamente até o nível $E$, se escrevem duas equações diferenciais, uma para calcular mudanças da massa do $P(\mathrm{~d} P / \mathrm{dt}=V-V=0)$, e a outra para o enriquecimento do $P\left(\mathrm{~d} P^{*} / \mathrm{dt}=V E-V P^{*} / P\right)$, com a solução do $P^{*}=P E[1-\exp (t V / P)]$. Num estudo da ERM do núcleo ${ }^{13} \mathrm{C}$, se faria uma infusão intravenosa do $S^{*}$, e ao longo de uma ou duas horas, se utilizaria a ERM para observar a aparência do $P^{*}$. Na análise dos dados, a concentração do $P$ é medida e se calcula o parâmetro $V$ que mais se adequa à equação exponencial construída com os dados obtidos. Enquanto esse exemplo ilustra

Figura 4 - Medidas de neurotransmissão pela ERM de ${ }^{13} \mathrm{C} .{ }^{26}$
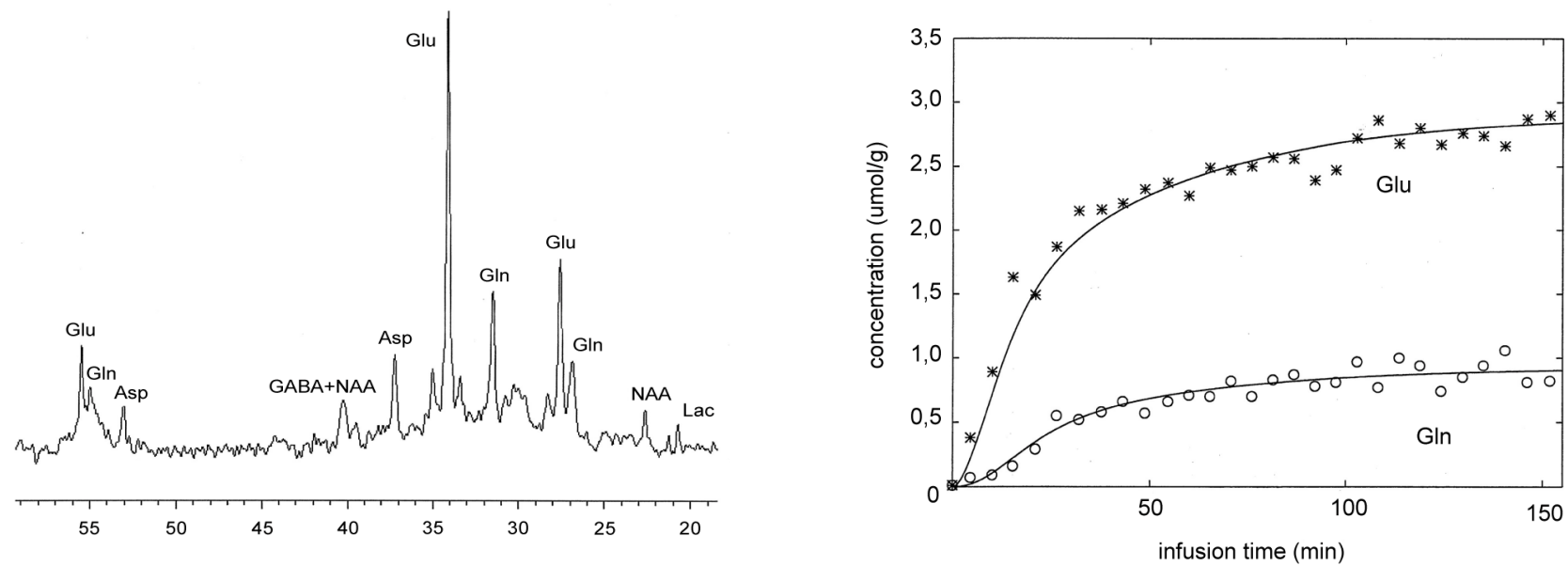

(A) Espectro adquirido in vivo do córtex occipito-parietal de um voluntário normal utilizando a detecção do ${ }^{13} \mathrm{C}$ num ímã de 2,1 Tesla, uma hora depois do começo da infusão de [1-13 Clglicose. Vê-se o ácido glutâmico, a glutamina, o GABA, o ácido aspártico e o ANA. (B) A aquisição de uma série de espectros durante uma infusão de [1-13 C]glicose provê o carbono ao ácido glutâmico e à glutamina, permitindo a obtenção de dados temporais para calcular as velocidades metabólicas e neurotransmissoras. As curvas sólidas representam as acumulações calculadas.

*Goddard A, Mason G, Almai A, Rothman D, Behar K, Petroff O, et al. Reductions in cortical GABA levels in panic disorder detected with 1Hmagnetic resonance spectroscopy. Arch Gen Psych 2000. No prelo. 
as idéias básicas do método, o esquema da neurotransmissão é bem mais complicado.

Para medir a velocidade da neurotransmissão, compara-se a acumulação do ácido glutâmico e da glutamina ao longo do tempo, pois estudos da histoquímica têm localizado a síntese da glutamina em astrócitos, enquanto a glutaminase é observada primariamente nos neurônios. ${ }^{24}$ Conseqüentemente, o ácido glutâmico é liberado dos neurônios, entra no astrócito, onde se converte em glutamina, e volta ao neurônio para se converter de novo no ácido glutâmico para a reutilização, tudo isto constituindo um ciclo neurotransmissor do glutamato-glutamina. Num estudo de ERM de ${ }^{13} \mathrm{C}$, o isótopo entra no cérebro através do transportador de glicose, é metabolizado via glicólise, entra no ciclo do ácido tricarboxílico, onde se incorpora no composto ácido alfa-ketoglutárico, que rapidamente é convertido em ácido glutâmico. Logo depois, é liberado pelos neurônios, passando pelos astrócitos na forma de glutamina. A acumulação do isótopo ao longo do tempo é mostrada na Figura 4B, a partir da qual pode-se calcular que no ser humano o glutamato é liberado para a neurotransmissão numa velocidade de $0,32 \mathrm{mmol}$ $\min ^{-1} \mathrm{~g}^{-1} \cdot{ }^{25}$ Estudos do mesmo processo em ratos têm demonstrado uma forte dependência da neurotransmissão glutamatérgica no nível basal de função cerebral, e se imagina que o mesmo aconteça no ser humano. Pesquisas dos transtornos psiquiátricos e do alcoolismo utilizando a ERM do ${ }^{13} \mathrm{C}$ estão começando agora e logo nos indicarão como são as ligações entre a função e a atividade glutamatérgica no ser humano.

\section{Discussão}

O futuro da ERM na psiquiatria pode avançar por três vias. Para conseguir aplicações gerais é preciso que a tecnologia e os equipamentos utilizados estejam mais amplamente disseminados, pois assim será prático estudar populações maiores. Essas populações mais diversas deverão incluir um grupo até agora muito pouco estudado em estudos de neuroimagem, qual seja o dos adolescentes, uma vez que a ERM não envolve radioatividade, o que aumenta consideravelmente a possível utilidade da ERM para esse grupo.

A segunda via será o desenvolvimento técnico continuado dos aparelhos de ERM, com maior resolução e possibilidades para novas aplicações. Isso já está acontecendo com rapidez, com novos métodos de imagens de ERM, provendo as concentrações não somente num único volume parcial, mas em várias regiões cerebrais. ${ }^{26}$ Além das imagens convencionais de ERM que são estáticas, estão se desenvolvendo agora também imagens de ERM que sejam cinéticas, utilizando o ${ }^{13} \mathrm{C} .{ }^{27}$ Um outro aspecto de importância crescente é que se considere nesses estudos a influência de variações em componentes cerebrais específicos, pois tecidos diferentes (substância cinzenta ou branca) freqüentemente contém concentrações químicas diferentes. ${ }^{28,29}$

A terceira via será o aumento dos campos magnéticos utilizados na clínica, de 1,5 Tesla para até 3 Tesla ou 4 Tesla. A amplitude do sinal e a resolução espectral aumentam de acordo com a amplitude do campo magnético, facilitando especialmente a mensuração do ácido glutâmico e da glutamina.

Em conclusão, é preciso que novos métodos e novos equipamentos sejam desenvolvidos, e que os métodos já desenvolvidos e testados sejam utilizados de maneira robusta e prática para pesquisas clínicas que envolvam populações maiores. Desta forma, pode-se antever o dia em que a ERM será uma ferramenta para diagnóstico em psiquiatria.

Trabalho realizado parcialmente com apoio financeiro das linhas de pesquisa NS37527, P30 MH30929-22, AA11321-01A2, NS34813 (Institutos Nacionais de Saúde, EUA); e da Theodore and Vada Stanley Foundation, da National Alliance for Research in Schizophrenia and Affective Disorders (NARSAD).

\section{Referências}

1. Mason G, Pan J, Ponder S, Twieg D, Pohost G, Hetherington H. Detection of brain glutamate and glutamine in spectroscopic images at 4.1T. Magn Reson Med 1994;32:142-5.

2. Urenjak J, Williams SR, Gadian DG, Noble M. Proton nuclear magnetic resonance spectroscopy unambiguously identifies different neural cell types. J Neurosci 1993;13:981-9.

3. Yue K, Rasgon N, Thomas M, Guze B, Fairbanks L, Curran J, et al. Cerebral metabolite changes across the menstrual cycle in women with PMDD and controls. A preliminary analysis. Proc Intern Soc Magn Reson Med 2000;1132.

4. Cendes F, Andermann F, Dubeau F, Matthews PM, Arnold DL. Normalization of neuronal metabolic dysfunction after surgery for temporal lobe epilepsy. Evidence from proton MR spectroscopic imaging. Neurol 1997;49:1525-33.

5. Bertolino A, Knable MB, Saunders RC, Callicott JH, Kolachana B, Mattay VS, et al. The relationship between dorsolateral prefrontal $\mathrm{N}$-acetylaspartate measures and striatal dopamine activity in schizophrenia. Biol Psychiatry 1999;45:660-7.
6. Bartha R, al-Semaan YM, Williamson PC, Drost DJ, Malla AK, Carr TJ, et al. A short echo proton magnetic resonance spectroscopy study of the left mesial-temporal lobe in first-onset schizophrenic patients. Biol Psychiatry 1999;45:1403-11.

7. Erecinska M, Silver IA. Metabolism and role of glutamate in mammalian brain. Prog Neurobiol 1990;35:245-96.

8. Sanacora G, Mason GF, Rothman DL, Behar KL, Hyder F, Petroff $\mathrm{OA}$, et al. Reduced cortical gamma-aminobutyric acid levels in depressed patients determined by proton magnetic resonance spectroscopy. Arch Gen Psychiatry 1999;56:1043-7.

9. Mason GF, Sanacora G, Anand A, Epperson N, Goddard A, Rothman D, et al. Distinct alterations in cortical amino acid levels in unipolar and bipolar depression assessed with MRS. Soc Neurosci 2000: Abstract 431.5.

10. Sanacora G, Mason G, Rothman D, Berman R, Zimolo Z, Krystal J. Cortical GABA concentrations are increased in depressed patients following treatment with selective serotonin reuptake inhibitors. Am Coll Neuropsychopharm 2000:276. 
11. Auer DP, Putz B, Kraft E, Lipinski B, Schill J, Holsboer F. Reduced glutamate in the anterior cingulate cortex in depression: an in vivo proton magnetic resonance spectroscopy study. Biol Psychiatry 2000;47:305-13.

12. Behar KL, Rothman DL, Petersen KF, Hooten M, Delaney R, Petroff OA, et al. Preliminary evidence of low cortical GABA levels in localized 1H-MR spectra of alcohol-dependent and hepatic encephalopathy patients. Am J Psychiatry 1999;156:952-4.

13. Epperson C, Mason G, Rothman D, Sanacora G, Krystal J. GABA dysregulation in premenstrual dysphoric disorder. Palestra proferida na Annual Meeting of the Society of Biological Psychiatry; 1999 Mai 14; Washington (DC).

14. Hetherington H, Pan J, Telang F, Pappas N, Volkow N. Reduced brain GABA levels in cocaine abusers. Proc Intern Soc Magn Reson Med 2000:523.

15. Mason GF, Behar KL, Lai JC. The $13 \mathrm{C}$ isotope and nuclear magnetic resonance: unique tools for the study of brain metabolism. Metabol Brain Dis 1996;11:283-313.

16. Fitzpatrick SM, Hetherington HP, Behar KL, Shulman RG. The flux from glucose to glutamate in the rat brain in vivo as determined by $1 \mathrm{H}$-observed, 13C-edited NMR spectroscopy. J Cereb Blood Flow Metabol 1990;10:170-9.

17. Mason GF, Rothman DL, Behar KL, Shulman RG. NMR determination of TCA cycle rate and a-ketoglutarate/glutamate exchange rate in rat brain. J Cereb Blood Flow Metab 1992;12:434-47.

18. Mason G, Pan J, Chu W-J, Newcomer B, Zhang Y, Orr R, et al. Measurement of the Tricarboxylic Acid Cycle Rate in Human Grey and White Matter in vivo by $1 \mathrm{H}-\{13 \mathrm{C}\}$ Magnetic Resonance Spectroscopy at 4.1T. J Cereb Blood Flow Metabol 1999;19:1179-88.

19. Pan JW, Mason GF, Vaughan JT, Chu WJ, Zhang Y, Hetherington HP. $13 \mathrm{C}$ editing of glutamate in human brain using J-refocused coherence transfer spectroscopy at 4.1 T. Magn Reson Med 1997;37:355-8.

20. Mason GF, Gruetter R, Rothman DL, Behar KL, Shulman RG, Novotny EJ. Simultaneous determination of the rates of the TCA cycle, glucose utilization, alpha-ketoglutarate/glutamate exchange, and glutamine synthesis in human brain by NMR. J Cereb Blood Flow Metabol 1995;15:12-25.
21. Sibson NR, Dhankhar A, Mason GF, Behar KL, Rothman DL, Shulman RG. In vivo 13C NMR measurements of cerebral glutamine synthesis as evidence for glutamate-glutamine cycling. Proc Natl Acad Sci USA 1997;94:2699-704.

22. Sibson NR, Mason GF, Shen J, Cline GW, Herskovits AZ, Wall JEM, et al. In vivo $13 \mathrm{C}$ NMR measurement of neurotransmitter glutamate cycling, anaplerosis and TCA cycle flux in rat brain during [2-13C]glucose infusion. J Neurochem 2000. No prelo.

23. Sibson NR, Dhankhar A, Mason GF, Rothman DL, Behar KL, Shulman RG. Stoichiometric coupling of brain glucose metabolism and glutamatergic neuronal activity. Proc Natl Acad Sci USA 1998;95:316-21

24. Shank R, Aprison M. Glutamate as a Neurotransmitter. In: Kvamme E, editor. Glutamate in Mammals. V. 2. Boca Raton: CRC; 1988. p. 3-20.

25. Shen J, Petersen KF, Behar KL, Brown P, Nixon TW, Mason GF, et al. Determination of the rate of the glutamate/glutamine cycle in the human brain by in vivo 13C NMR. Proc Natl Acad Sci USA 1999; $96: 8235-40$.

26. Posse S, Tedeschi G, Risinger R, Ogg R, Le Bihan D. High speed $1 \mathrm{H}$ spectroscopic imaging in human brain by echo planar spatialspectral encoding. Magn Reson Med 1995;33:34-40.

27. Hyder F, Renken R, Rothman DL. In vivo carbon-edited detection with proton echo-planar spectroscopic imaging (ICED PEPSI): [3,4-(13) $\mathrm{CH}(2)]$ glutamate/glutamine tomography in rat brain. Magn Reson Med 1999;42:997-1003.

28. Hetherington HP, Pan JW, Mason GF, Adams D, Vaughn MJ, Twieg DB, et al. Quantitative $1 \mathrm{H}$ spectroscopic imaging of human brain at $4.1 \mathrm{~T}$ using image segmentation. Magn Reson Med 1996;36:21-9.

29. Pfefferbaum A, Adalsteinsson E, Spielman D, Sullivan EV, Lim KO. In vivo spectroscopic quantification of the $\mathrm{N}$-acetyl moiety, creatine, and choline from large volumes of brain gray and white matter: effects of normal aging. Magn Reson Med 1999;41:276-84.

\section{Correspondência: Graeme F. Mason}

Magnetic Resonance Center, Yale University, School of Medicine

333 Cedar St. - 06520-8043 New Haven, CT, USA

Tel.: (00xx11) (203) 737-1478 - Fax: (00xx11) (203) 785-6643 - E-mail: graeme.mason@yale.edu 